\title{
Hierarchical Genetic Algorithms for composite laminate panels stress optimisation
}

\author{
P. Isasi \& A. Sanchis \& J.M. Molina \& A. Berlanga \\ Departamento de Informática \\ Universidad Carlos III de Madrid \\ 28911, Leganés, Madrid \\ E-mail: isasi@ia.uc3m.es
}

\begin{abstract}
Genetic Algorithms (GA's) have demonstrated to be a powerful technique for solving optimisation problems. An adaptation of a simple GA to optimise laminate design is presented in this paper.

This problem has special features that makes it different from traditional problems in which GA's have been applied. In this case, there is not a single function to optimise, by the opposite we have to the solutions must try to optimise two parameters, both resistance and number of plies. These characteristics make the problem a multi-objective optimisation one. Symmetry and equilibrate constrains have also been included in the solution. A new perspective to solve this problem using GA's techniques has been introduced.
\end{abstract}

\section{INTRODUCTION}

The design of laminated composite panels could be seen, some times, as a continuous optimisation problem. In this problem, the variables to optimise are the thickness of laminates and the orientation angle between them [9]. However, in most of practical problems, the laminate thickness is kept constant, and angles vary over a small set of alternatives, usually 0,90 and 45 degrees. In these terms, the design of laminated becomes an optimisation problem where only plies sequence is considered.

Genetic Algorithms (GA's) are stochastic techniques for searching problems, based on genetic population ideas [3]. GA's work with populations of individuals. Each individual represents a hypothetic solution (design). The recombination of the most desirable features of each existing design guides the search toward an optimal design. This search follows the ideas of evolution in Darwin theory. First, some members of the population are selected, favouring the best members of the population and to guarantee that the best features could be transmitted to generate new, and probably better, designs. In this process, new designs are created and tested. GA's do not use any gradient information for the searching process, and are not sensible to the design space complexity.

Holland [4], Rechenberg [8], Rosenberg and Goldberg [2] were the first researchers in using the GA's as optimisation technique, since then GA have been applied to commerce, engineering mathematics, medicine and pattern recognition with hopeful results [10]. Recently, in some works GA's have been applied efficiently in structures design $[5,7,6]$.

GA's have a combinatorial nature because they work more with a codification of the variables of the design rater than with the variables them-self. This become GA's computationally able of dealing with continuous design variables by specifying the exact precision required.

In this article, the problem of optimising the number of plies and their stacking sequence in the design of laminated composite panels is considered. This problem has some peculiarities that makes inapplicable the usual GA technique. Thus, a modification of the canonical GA is needed as is described in the next section.

\section{GENETIC ALGORITHMS}

In GA's the design variables are codified in fixed length strings, analogous to the chromosomes in biologic systems. The strings elements (usually in binary code, but formed here by quartets) are the analogy of genes. Each string represents a design. GA's are iterative procedures, where the size of a population of possibles designs, or candidates designs, is kept constant. Each step in the iterative process is called a generation. The initial set of designs, randomly generated, is called initial population.

GA's imitate the process of evolution by means of Natural Selection to solve optimisation problems. This is a Darwinian approach to the idea of the survival of the fittest. However, in a natural process of selection, individuals have to compite in order to overcome the survival chances of others. In computational terms this has to be measured in terms of an evaluation function called fitness function. This function produces a fitness value for each individual and it is used for the selection 
process. After selecting the parents, new designs are created applying some functions to those parents. This functions are called genetic operators: mutation, inversion and crossover, to create new designs, in the hope that this new designs achieved improve features with respect to old ones. New individuals replace those (the old ones) in the old population to create a new population for the next generation.

The components of the GA are:

- Individuals.- Each partial solution to a problem is codified in a string. Each string is called an individual. In this case each individual is a laminate design. For instance, the laminate $\left[0_{4}, \pm 45_{6}, 0_{2}, 90_{2}\right]_{s}$ is an individual.

- Population.- A set of individuals is called a population. The GA method consists in generating new populations of individuals in such a way that better individuals could be generated in newer populations.

- Genetic Operators.- GO are different functions applied to populations to generate new populations. The most used are: Selection, Crossover and Mutation.

- Fitness function.- FF is a measure of the goodness of an individual, in terms of the ability of this individual to solve the problem. This measure is used as a parameter for the GO and guides the generation of new better populations.

The general optimisation procedure followed by a $\mathrm{GA}$ is:

1. To generate randomly an initial population.

2. To apply the GO to the population in time " $t$ " to generate the population in " $t+1$ ".

3. Repeat step 2 until some convergence criteria could be accomplished (for instance the variability of the population reduces some fixed threshold).

This method assures that the average of the fitness function for all the population does not decrease with time [4].

\section{PROBLEM DESCRIPTION}

The problem is to find an optimal design of a composite laminate. The variables involved in the optimisation are the ply thicknesses and ply orientations. For many practical problems ply thicknesses are fixed and ply orientations are limited to a small set of angles such as 0,90 , and $+/-45 \mathrm{deg}$. The final design achieved is the number and orientation of plies that resist some specific strength constraints.

The searched designs have to be well balanced, symmetric and with a variable number of plies. As no all possible designs are viable, the GA must generate only the valid ones. So, it is necessary to introduce some restrictions in the GA. The above characteristics can be reached in two ways: designing "ad-hoc" genetic operators, or codifying the problem in such a way that only valid solutions could be generated. In the first case, it is necessary to know if the new operators are able to keep the efficiency and generalisation level. Most of the non general purpose genetic operators reduces the GA's efficiency, making bigger the search space, or introducing redundancy in the solutions [1]. In this case, a special codification able to include the above restrictions has been designed.

Each design is codified by a four base string. The meaning is the following:

\begin{tabular}{|l|l|}
\hline 0 & A 0 degrees ply \\
\hline 1 & A 90 degrees ply \\
\hline 2 & two plies of 45 and -45 degrees respectively \\
\hline 3 & No ply in this position \\
\hline
\end{tabular}

In one string, only the first half of the laminate is represented in the proposed codification. This is due to the symmetry restriction, the second half of the laminate can be rebuilt from the first half. The maximum laminated size is kept constant (in this case is set to a value of 20 ). However the value 3 in the above codification allows variable sized solutions. For individuals of size 20 the maximum possible number of plies is 80 , this maximal plies individual is:

$$
\text { [22222222222222222222] }
$$

that corresponds whit with the following design:

$$
\left[ \pm 45_{20}\right]_{s}
$$

With this codification, for instance, the individual:

$$
\text { [13212210033033012031] }
$$

will be transformed, removing gaps (empty space), into the design:

$$
\left[90, \pm 45,90, \pm 45_{2}, 90,0_{4}, 90, \pm 45,0,90\right]_{s}
$$

This design has 36 plies, 10 of 0 degrees, 10 of 90 degrees, 8 of 45 degrees and 8 of -45 degrees (figure 1).

Another important feature of the composite laminate problem is that not only one function has to be optimised. A good design has to be firstly secure. This means that the laminate has to resist from some specific configuration of forces, to the failure. Once the above restriction is assured, the good designs are the cheapest ones. This objectives could be resumed in designing a laminate with less of the plies as possible that assure the resistance laminate from a specific configuration of forces. Many optimal solutions could be 
$[33233302313311333033]$

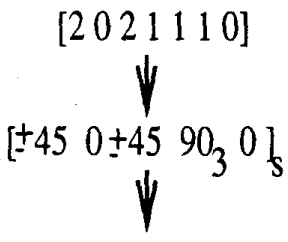

$[45-45045-4590909000909090-45450-4545]$

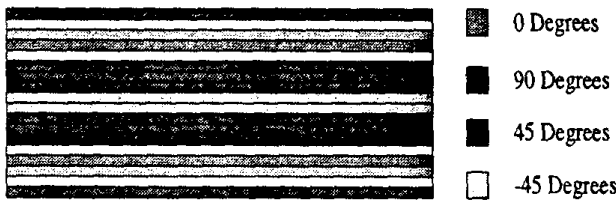

Figure 1: A design codification in a four base string

reached following the above criteria: "equal thickness with no failure". In this case, is important to kept the laminate as far as possible of the failure. This could be measured with the stress values $(r)$. The preferred configuration is those with shorter $r$ 's values.

The fitness function in the GA has been modified to include the multi-criteria evaluation of the designs. For this purpose, the fitness function has been divided in two independent functions:

- The first one is only a computation of the number of plies.

- The second one is a value that estimate the distance to failure of the design.

This computation is done by computing the average of stress values. In this way the GA is modified becoming an optimisation process of two different hierarchical functions.

To implement the multi-objective hierarchical fitness function, some variations in the tournament selection method have been included. The tournament selection consists in the uniform selection of some few individuals from the population constituting the tournament set. Then a single individual is selected from the tournament set following fitness criteria, the individual with a best fitness value is selected, and included in the mating pool. The mating pool constitutes the set of individuals that will participate in the crossover process to generate the next generation of individuals.

In this case, each individual has two independent fitness values, one related with the number of plies, and the other related with the stress values. The procedure for tournament selection is now as follows:
1. Generate the tournament set randomly.

2. Select from this set the individual with best fitness value considering the first fitness value.

3. If only one individual has the best value for the first fitness, include this individual in the mating pool.

4. If more than one individual have identical and maximal value for the first fitness value, select among theirs the individual with higher second fitness value.

This procedure can be generalised for any number of fitness functions (Figure 2).

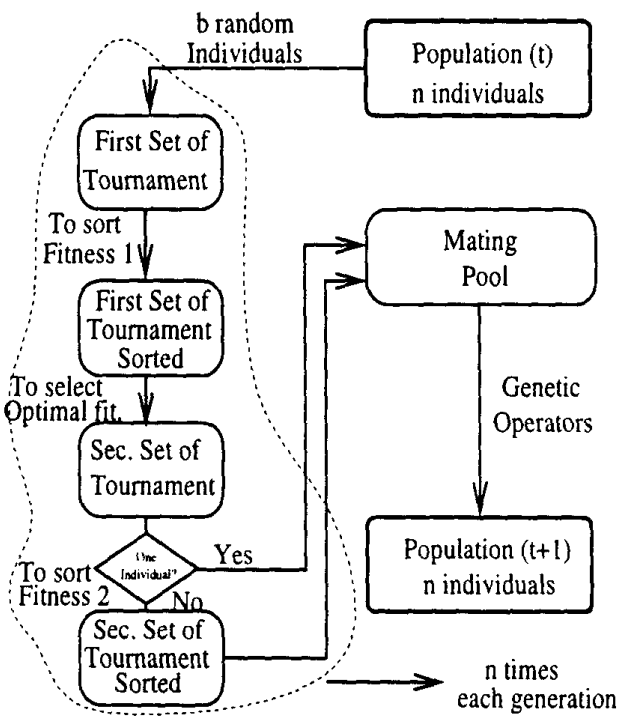

Figure 2: Genetic Algorithm with hierarchical fitness functions.

\section{RESULTS}

The above algorithm has been applied to the optimisation of a composite laminate of a simply supported rectangular panel under in-plane loads using the Tsai Hill failure criteria. The material used in this work correspond to T300/5208, with the following data:

- Elastic constants (GPa): Ex, Ey, Es= 3D 181, $10.3,7.17 ; \mathrm{u}=3 \mathrm{D} 0.28$

- Resistence values (MPa): X, X', Y, Y',S=3D 1500 , $1500,40,246,68$

The achieved solutions correspond with the optimisation under a system of loads with only a couple of loads $\left(N_{x}, N_{y}, N_{x y}\right)=3 D(-7,1,2) M N / m$.

As it could be expected, under this system of loads, the results corresponding to same number of plies with 
the same orientations but different ordering, are identical. The final parameters used are: simple crossover with probability 1.0 , mutation with probability 0.05 , population size 100 , elitist overlapping size 0.01 . Many experiments have been done, with different parameters, before the above values have been fixed. The method has been demonstrated no to be sensible to the variation in the parameters. Some other multi-optimisation and sharing options have been tested, but the performance of the hierarchical mechanism designed has been demonstrated the best mechanism for this problem.

In the table some achieved designs are shown, corresponding to different periods in the evolution of the best solution.

\begin{tabular}{|r|r|r|r|r|r|r|}
\hline Step & $\mathbf{0}^{\circ}$ & $\mathbf{9 0}^{\circ}$ & $\mathbf{4 5}^{\circ}$ & $\mathbf{- 4 5}^{\circ}$ & Plies & Stress \\
\hline 25 & 10 & 8 & 4 & 4 & 26 & 0.049 \\
\hline 50 & 4 & 16 & 2 & 2 & 24 & 0.078 \\
\hline 75 & 6 & 10 & 2 & 2 & 20 & 0.130 \\
\hline 100 & 10 & 4 & 2 & 2 & 18 & 0.554 \\
\hline
\end{tabular}

\begin{tabular}{|c|c|}
\hline Step & Solution \\
\hline 25 & {$\left[0 \pm 450_{4} 90_{4} \pm 45\right]_{s}$} \\
\hline 50 & {$\left[\begin{array}{llllll}90_{3} & 0 & \pm & 90_{2} & 0 & 90_{3}\end{array}\right]_{s}$} \\
\hline 75 & {$\left[\begin{array}{llll} \pm 45 & 90 & 0_{3} & 90_{4}\end{array}\right]_{s}$} \\
\hline$\overline{100}$ & {$\left[\begin{array}{llllll}0 & \pm 45 & 0_{2} & 90_{2} & 0_{2}\end{array}\right]_{s}$} \\
\hline
\end{tabular}

Table 1: Designs evolution through simulation.

At the end of the process, some solutions with identical number of plies have been achieved. All those solutions are valid, and can be distinguished by the average value of the stress. In table some final solutions are shown. The solutions are sorted by the average value of the stress. In this ordering the first solutions are farther away from the failure than the last ones.

The ten solutions in table can be grouped in five classes, depending on the number of plies of each orientation in the solution. The farest solution from failure uses 4, 6, 4 and 4 plies with orientations 0, 90, 45 and -45 degrees respectively. For other solutions, not only the number of plies for each orientation but the sortering of each ply, is achieved. These results allow a deep analysis of the solutions. In this specific case, it can be said that better solution uses a composition of $45^{\circ}$ and $90^{\circ}$ Iaminates more than other orientations. All the solutions that not follow this rule result in a worse design.

\section{CONCLUSIONS}

A Genetic Algorithm has been developed and verified for searching and optimising a laminate design. This method has been proved to be powerful for this task, and easily handle the discrete nature of the stacking sequence design problems. It can be easily enforce constrains thar are difficult to handle with continuous

\begin{tabular}{|r|r|r|r|r|r|}
\hline $\mathbf{0}^{\circ}$ & $\mathbf{9 0}^{\circ}$ & $\mathbf{4 5}^{\circ}$ & $\mathbf{- 4 5}^{\circ}$ & Plies & Stress \\
\hline 4 & 6 & 4 & 4 & 18 & 0.188 \\
\hline 6 & 8 & 2 & 2 & 18 & 0.249 \\
\hline 6 & 8 & 2 & 2 & 18 & 0.249 \\
\hline 6 & 8 & 2 & 2 & 18 & 0.249 \\
\hline 6 & 8 & 2 & 2 & 18 & 0.249 \\
\hline 8 & 6 & 2 & 2 & 18 & 0.392 \\
\hline 8 & 6 & 2 & 2 & 18 & 0.392 \\
\hline 10 & 4 & 2 & 2 & 18 & 0.554 \\
\hline 10 & 4 & 2 & 2 & 18 & 0.554 \\
\hline 8 & 2 & 4 & 4 & 18 & 0.561 \\
\hline
\end{tabular}

\begin{tabular}{|c|}
\hline Solution \\
\hline$\left[ \pm 450 \pm 4590_{3} 0\right]_{s}$ \\
\hline$\left[\begin{array}{lllllll}90 & \pm 45 & 0_{2} & 90_{2} & 0 & 90\end{array}\right]_{s}$ \\
\hline$\left[\begin{array}{llll} \pm 45 & 0_{3} & 90_{4}\end{array}\right]_{s}$ \\
\hline$\left[ \pm 4590_{2} 0900900\right]_{s}$ \\
\hline$\left[900_{3} \pm 4590_{3}\right]_{s}$ \\
\hline$\left[\begin{array}{llllllllll}0 & \pm & 0_{2} & 90_{2} & 0 & 90\end{array}\right]_{s}$ \\
\hline$\left[\begin{array}{lllllllll}0 & \pm & 0_{2} & 90 & 0 & 90_{2}\end{array}\right]_{s}$ \\
\hline$\left[\begin{array}{lllllll}0 & \pm 45 & 0_{2} & 0_{2} & 90_{2} & 0_{2}\end{array}\right]_{s}$ \\
\hline$\left[\begin{array}{llll} \pm 4 & 0_{2} & 90_{2} & 0_{3}\end{array}\right]_{s}$ \\
\hline $\pm 450 \pm 450900_{2}$ \\
\hline
\end{tabular}

Table 2: Final result from one simulation.

optimisation. It is also easy to introduce some requirements as to optimise both the tickness of the design and the distance to the failure.

The designed hierarchical fitness function has proved its ability to optimise more than one goal at once, being very useful in multi-optimisation problems.

The codification and achievement of more than one design can help in the discovery of rules that implicitly governs the features of the designs. This is very useful for the designers to know the possibilities and characteristics of good designs. Furthermore the GA can yield many designs with comparable performance for the designer to choose from.

\section{REFERENCES}

[1] K. DeJong. Learning with genetic algorithms: An overview. Maching Learning, I(3):121-138, 1988.

[2] D.E. Goldberg. Optimal initial population size for binary coded genetic algorithms. Technical Report TCGA Rept. 85001, Univ. de Alabama, Tuscaloosa, AL, 1985.

[3] D.E. Goldberg. Genetic Algorithms in search optimization and machine learning. Addison-Wesley, Reading, MA., 1989.

[4] J. Holland. Adaptation in Natural and Artificial Systems. Univ. de Michigan Press, Ann Arbor, MI, 1975 . 
[5] S. Nagendra. Design of a blade stiffened composite pannel by a genetic algoritm. In 34TH AIAA/ASME/ASCE/AHS/ASC Structures, Structural Dynamics and Materials Conference, pages 2418-2436, Washington DC, USA, April 1993. AIAA.

[6] S. Nagendra, R.T Haftka, and Z Gurdal. Staking sequence optimization of simply supported laminates with stability and strain constraints. AIAA Journal, 30(8):2132-2137, August 1992.

[7] H. Nanfang. Tabu search method with random moves for globally optimal design. International Journal for Numerical Methods in Engineering, 35(5):1055-1070, September 1992.

[8] J. Rechenberg. Cybernetic solution path of an experimental problem. Technical Report Library translation 1122, Royal Aircraft Establishment, Farnborough, England, UK, 1965.

[9] L.A. Schmit and B. Farshi. Optimum design of laminate fibre composite plates. International Journal for Numerical methods in Engineering, 11(1):623-640, 1977.

[10] S.J. Wu and P.T. Chow. Integrated discrete and configuration optimization of trusses using genetic algorithms. Computer and Structures, 55(1):695$702,1995$. 